\title{
Comunicación científica de archivística en España sobre temas de tecnología digital (2001-2018)
}

\section{Scientific communication related to digital technology topics in archival science in Spain (2001-2018)}

\author{
Cynthia Roncaglio; Concepción Mendo-Carmona
}

Cómo citar este artículo:

Roncaglio, Cynthia; Mendo-Carmona, Concepción (2020). “Comunicación científica de archivística en España sobre temas de tecnología digital (2001-2018)". Profesional de la información, v. 29, n. 4, e290434.

https://doi.org/10.3145/epi.2020.jul.34

Artículo recibido el 24-02-2020

Aceptación definitiva: 08-06-2020

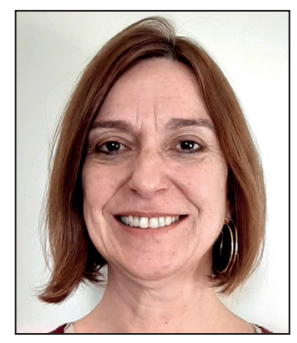

Cynthia Roncaglio $\square$

https://orcid.org/0000-0002-4859-4296

Universidade de Brasília

Faculdade de Ciência da Informação

Campus Universitário Darcy Ribeiro

Gleba A - Asa Norte, Brasília DF

70910-900, Brasil

cynthia.roncaglio@gmail.com

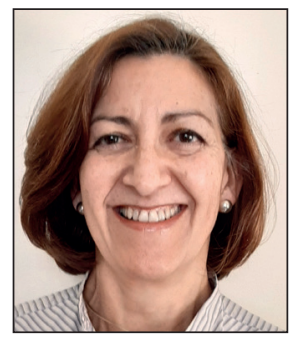

Concepción Mendo-Carmona https://orcid.org/0000-0003-3736-2649

Universidad Complutense de Madrid

Facultad de Ciencias de la Documentación Santísima Trinidad, 37

28010 Madrid, España

cmendo@pdi.ucm.es

\section{Resumen}

Entre 2001 y 2018, el período que abarca esta investigación, se percibe a través de las publicaciones científicas sobre archivística en España que el tema de la tecnología digital está cada vez más presente en las reflexiones teóricas, prácticas y opciones metodológicas archivísticas. A partir de esta observación, el objetivo del artículo es identificar el alcance de la interrelación temática entre archivos, archivística y tecnología digital. La investigación es de naturaleza exploratoria, descriptiva y analítica, basada en un análisis cuantitativo y cualitativo de artículos científicos disponibles en las revistas Tábula, Lligall y Boletín de la Anabad, publicados de 2001 a 2018, luego organizados por categorías de análisis. Los resultados en términos cuantitativos indican que la categoría más destacada es la que llamamos "Influencias mutuas entre archivos, archivística y tecnología digital". La categoría que ocupa el segundo lugar es "Relatos de experiencias", en cuanto a las otras, "Relaciones entre archivos, archivística y tecnología digital", y "Retos teóricos y prácticos" se sitúan casi en el mismo nivel, aunque muy por detrás de las dos primeras categorías. Además, se concluye que hay una tendencia en la archivística actual a experimentar las posibilidades tecnológicas para su desarrollo más que innovar en términos teóricos y epistemológicos.

\section{Palabras clave}

Archivos; Archivística; Tecnología digital; Tábula; Revista Lligall; Boletín de la Anabad; Revistas; España; Teoría; Epistemología; Metodologías.

\begin{abstract}
Between 2001 and 2018, the period covered by this research, it is perceived through the scientific publications on Archival Science in Spain that the topic of digital technology is increasingly present in the theoretical reflections, practices, and archival methodological options. Based on this observation, the objective of this article is to identify the scope of the thematic interrelations between archives, archival science, and digital technology. The research is exploratory, descriptive, and analytical in nature, based on a quantitative and qualitative analysis of scientific articles available in the Tábula, Lligall, and Boletín de la Anabad journals, published from 2001 to 2018, and organized by categories. The results in quantitative terms indicate that the most prominent category is what we call "Mutual influences between archives, archival science, and digital technology." The category that occupies the second place is "Description of experiences," followed by "Relations between archives, archival science, and digital technology" and "Theoretical and practical challenges" at
\end{abstract}

Financiación

Investigación financiada por la Fundação de Apoio à Pesquisa do Distrito Federal (FAP/DF), Brasil. 
almost the same level, albeit far behind the first two categories. In addition, it is concluded that there is a tendency in current archiving to explore the technological possibilities for its development rather than innovate in theoretical or epistemological terms.

\section{Keywords}

Archives; Archive Science; Archival Science; Archivistics; Archival; Digital technology; Journals; Tábula; Revista Lligall; Boletín de la Anabad; Spain; Theory; Epistemology; Methodology.

\section{Introducción}

Una constante en la historia de la archivística es que su evolución ha estado vinculada a las necesidades planteadas por los avances tecnológicos de las sociedades, tanto en lo que se refiere a la capacidad de generación de documentos como a la importancia jurídica que a estos se les ha atribuido, en particular a su relevancia probatoria.

Estados Unidos siempre ha estado a la vanguardia de la innovación tecnológica, pero actualmente países como Corea del Sur, Japón o China se han convertido en verdaderas potencias en este aspecto; por su parte, la Unión Europea está trabajando también decididamente en las tecnologías de la información y la comunicación, potenciando proyectos que incluyen su aplicación a los archivos.

Asimismo, hay que resaltar que, a diferencia de otros aspectos de la evolución de la archivística en los que la iniciativa la llevó el Consejo Internacional de Archivos (CIA) y gracias a él se consiguieron importantes logros archivísticos, el desarrollo experimentado en los últimos tiempos en el campo de las tecnologías se debe a las investigaciones realizadas en el entorno universitario, debido fundamentalmente al establecimiento definitivo de la enseñanza de esta ciencia en la universidad en casi todos los países.

Aunque el avance más espectacular corresponde a las innovaciones de los últimos años, la tecnificación y automatización de las tareas archivísticas es ya patente en 1972, durante el Congreso Internacional de Archivos celebrado en Moscú, con la creación de la Comisión Internacional de Informática y su transformación posteriormente en dos comités: el Comité sobre documentos electrónicos y el Comité sobre informática en los archivos, creados en el Congreso Internacional de Montreal (1994). Otra manifestación de su inquietud por el tema es el hecho de que dedicara, desde finales de la década de los ochenta, al menos una sesión de todos sus congresos internacionales a las incidencias de las tecnologías en los archivos.

Desde los primeros proyectos y aplicaciones informáticas hasta la situación actual se observan unas etapas claras en el proceso evolutivo. En un primer momento, el interés radicó en permitir el acceso a los fondos documentales por medio de la automatización o mecanización de los procesos descriptivos en colecciones o fondos cerrados. La aplicación de las tecnologías ópticas también posibilitó la creación de aplicaciones dirigidas a preservar el rico patrimonio documental que conservan los archivos, como alternativa a las técnicas de microfilmación, y a ofrecer un más amplio y mejor servicio a la cultura y la investigación.

Sin embargo, la revolución experimentada en los últimos tiempos en las tecnologías se debe a la imparable transformación digital en la que el mundo se encuentra inmerso. El reto de la archivística del siglo XXI es el tratamiento, conservación y acceso de los documentos en soporte digital. El desplazamiento del documento en papel por el documento electrónico en todos los campos de la sociedad y las implicaciones que este cambio conlleva en los archivos, plantea constantes desafíos a la archivística.

Entre 2001 y 2018, período cubierto por esta investigación, está claro en la comunicación científica nacional y extranjera que el uso generalizado y consolidado de la tecnología digital influye cada vez más en las reflexiones teóricas, las prácticas y las opciones metodológicas archivísticas. Dado este hallazgo, y considerando que esta investigación es parte de un enfoque más amplio de las interrelaciones entre archivos, archivística y tecnología digital en Brasil y España, el objetivo de este trabajo es identificar y analizar revistas científicas que aborden este tema en España. Nos preguntamos, en el contexto de la producción científica española, ¿cuáles son los subtemas que han despertado el interés de la comunidad profesional y científica que escribe y difunde artículos sobre el tema? y ¿qué dicen estos temas sobre el desarrollo de la actual archivística en España?

Mediante una metodología exploratoria, analítica y descriptiva se realiza un análisis de parte de la producción científica sobre el tema: archivística y tecnología digital en España, durante los primeros dieciocho años del siglo XXI, centrándose en un análisis cuantitativo y cualitativo de los artículos publicados en las siguientes revistas científicas españolas en el campo de la archivística y áreas afines:

- Tábula (Castilla y León);

- Revista Lligall (Cataluña);

- Boletín de la Anabad (España). 
La selección de las revistas citadas se debe a su larga trayectoria, al hecho de que incluyen varios temas relacionados con los archivos y la archivística, razón por la cual varios autores respetados y reconocidos en el campo están presentes y representan la archivística en España, siendo el Boletín de la Anabad la única revista de ámbito nacional. También hay contribuciones de autores extranjeros en las tres revistas, pero en menor número, como se verá a continuación.

La comunicación científica es fundamental para la difusión y desarrollo de investigaciones y experiencias, porque a través de ellas es posible conocer y debatir los resultados parciales y finales de la investigación científica, hacer reflexiones sobre la práctica y la teoría, a través de ensayos y relatos que vigorizan y permiten la elaboración de nuevas investigaciones y debates. Se entiende que analizar la comunicación tecnocientífica de los archivos y la archivística también ayuda a comprender el estado del arte y a estimular la discusión sobre nuevos campos de investigación necesarios para su desarrollo.

\section{Aproximaciones entre archivística e informática en España}

Los inicios de la aplicación de la informática en los archivos españoles se sitúa en la década de los años 70 del siglo pasado. Desde aquellos orígenes hasta el momento actual se pueden identificar dos etapas:

- una primera centrada en la aplicación de tecnologías en el tratamiento de los archivos en papel;

- una segunda, más reciente, caracterizada por buscar soluciones conjuntas para la gestión documental en el campo de la administración electrónica y su posterior organización archivística.

A su vez, en la primera etapa se suceden tres fases en las que se llevan a cabo proyectos y actividades que involucran la tecnología digital:

- informatización del proceso descriptivo;

- creación de sistemas de gestión integral de archivos;

- archivos en red.

Los primeros proyectos centraban su atención en la aplicación de la informática al proceso descriptivo y de recuperación de la información. El objetivo era la descripción de un fondo específico o la realización de un instrumento descriptivo determinado. El avance de las tecnologías de la información y de la comunicación permitió crear sistemas únicos y estructurados de información archivística relacionando distintos niveles de descripción y acceder a la información por distintas vías. Junto a ello, la tecnología óptica, es decir, la combinación del disco óptico como soporte de almacenamiento y técnicas de reconocimiento óptico de caracteres (OCR), posibilitaron la realización de proyectos informáticos de preservación y difusión del patrimonio documental cada vez más sofisticados. Desde esta perspectiva, el avance más significativo se debió a la convergencia de la informática, la tecnología digital y las telecomunicaciones, que posibilitaron la creación de programas de difusión y acceso al patrimonio documental (Sánchez-Mairena, 2014).

La tecnología se aplicaba sobre todo en los archivos históricos o de fondos cerrados, pero no se abordaba de forma global el tratamiento archivístico en instituciones administrativas. A partir de aquellas experiencias se han ido elaborando sistemas para el seguimiento y control de los documentos administrativos a lo largo de su tramitación y su posterior clasificación y descripción mediante programas de gestión integral de archivos que funcionan conectados con las unidades administrativas productoras.

La segunda etapa en la vinculación entre los archivos, la archivística y la informática en nuestro país, está determinada por la implantación de la administración electrónica bajo las directrices impuestas por la Unión Europea. Los primeros objetivos del e-gobierno se centraban en lograr la eficiencia del sector público en el desempeño de servicios dirigidos a los ciudadanos, principalmente en educación y comercio electrónico. A lo largo de estos años el gobierno electrónico ha avanzado hasta la consideración de que la participación ciudadana es imprescindible, como coproductora de los servicios públicos y para garantizar la transparencia y responsabilidad de los pode-

La tecnología se aplicaba sobre todo en los archivos históricos o de fondos cerrados, pero no se abordaba de forma global el tratamiento archivístico en instituciones administrativas

res públicos en el fortalecimiento de las democracias. En este recorrido se han dado importantes pasos para conseguir la seguridad digital, tales como la sustitución del documento en papel por el electrónico, la identificación ciudadana, la firma electrónica y la interoperabilidad, lo que ha planteado nuevos desafíos a la archivística.

Como consecuencia de lo expuesto, una de las transformaciones operadas en la disciplina ha sido su enorme sujeción a la normativa, entendiendo por esta el conjunto de reglas jurídicas de obligado cumplimiento, así como aquellas otras especificaciones técnicas, de observancia no obligatoria, que aprueba un organismo de normalización. Además de los estándares técnicos publicados por la Organización Internacional de Normalización (ISO) para la gestión de documentos, en España desde los inicios del siglo XXI la legislación ha ido creando el marco jurídico adecuado para garantizar que los documentos digitales cumplieran con las mismas garantías legales que el soporte tradicional. Primero fueron las leyes de firma electrónica, más tarde la derogada ley de acceso electrónico de los ciudadanos a los servicios públicos, y los reales decretos de interoperabilidad y seguridad nacional. A ellos se ha sumado, en los diez últimos años, otro considerable 
número de leyes conteniendo normas técnicas de interoperabilidad para la administración electrónica que abarcan la gestión, los metadatos, el documento electrónico, el expediente electrónico, los estándares, la digitalización de documentos, la firma y sello electrónicos, protocolos de intermediación de datos, relación de modelos de datos, política de gestión de documentos electrónicos... La Ley 39/2015, de 1 de octubre, del procedimiento administrativo común de las administraciones públicas obliga a las administraciones a prescindir del papel sustituyéndolo por soporte digital, y establece en el art. 17, que

"cada Administración deberá mantener un archivo electrónico único de los documentos electrónicos que correspondan a procedimientos finalizados, en un formato que permita garantizar la autenticidad, integridad y conservación del documento, así como su consulta con independencia del tiempo transcurrido desde su emisión" (España, 2015) ${ }^{1}$.

Desde todos los ámbitos territoriales, estatal, autonómico y local se han implantado proyectos para potenciar el archivo, puesto que la administración electrónica ha revalorado su importancia, un tanto olvidada, para el correcto funcionamiento de las instituciones como custodio de actos administrativos y como garante de los derechos democráticos.

Así pues, ante la radical transformación de los archivos y de los documentos que custodian, la archivística española de los últimos tiempos ha ido reflexionando y elaborando trabajos científicos para contribuir a la evolución de la disciplina de acuerdo con las nuevas exigencias de la sociedad.

\section{Metodología}

En el contexto de las transformaciones tecnológicas, de los archivos y de la archivística como disciplina científica, la presente investigación consiste en una encuesta cualitativa y cuantitativa en tres revistas seleccionadas, utilizando palabras clave para recuperar artículos acerca de archivos y archivística relacionados con la tecnología digital. Las palabras clave elegidas fueron combinaciones de los términos: archivos, archivística, documentos electrónicos, documentos digitales, tecnología digital.

Tras este paso, los metadatos previamente definidos (año/semestre, número, tema -de la revista, si corresponde-), autor (es), título, resumen, palabras clave, referencias) se transfirieron a una hoja de cálculo para su posterior análisis, utilizando categorías de análisis diseñadas para este propósito.

\section{Tábula: estudios archivísticos de Castilla y León https://www.acal.es/index.php/tabula}

Se publica anualmente desde 1992 por la Asociación de Archiveros de Castilla y León $(A C A L)$, dedicada a la investigación de la teoría y la práctica archivística. Cada número aborda monográficamente un tema de interés archivístico. El acceso al texto completo está restringido a los asociados o a quien compre la revista. En la biblioteca de la Facultad de Ciencias de la Documentación de la Universidad Complutense de Madrid, donde se realizó la recogida de datos, está disponible la versión impresa de los años 2002 a 2018. En la web de la ACAL, la versión electrónica está disponible de 2005 a 2018, pero está incompleta. En el portal Dialnet hay un sumario completo disponible de los años 2002 a 2018; sólo en unos pocos años aparece el resumen. Es posible que no se haya publicado un número en 2001, ya que no se encontró ni la versión impresa ni electrónica del mismo.

\section{Revista Lligall}

https://arxivers.com/publicacions/revista-lligall

Es una publicación científica anual de la Associació d'Arxivers - Gestors de Documents de Catalunya (AAC-GD). Lligall significa legajo. Publicada desde 1988 , incluye los principales debates y contribuciones sobre teoría y práctica archivística en el país. La publicación consiste en un dossier central que recopila información sobre cuestiones de archivo y mundo archivístico, contribuciones o noticias importantes del grupo de archiveros catalanes sobre temas candentes. Hasta 2017 el acceso al texto completo está restringido. Algunos artículos tienen resúmenes y palabras clave y otros no. Para este estudio utilizamos las versiones impresas y en $C D$ disponibles en la biblioteca de la Facultad de Ciencias de la Documentación de la Universidad Complutense de Madrid.

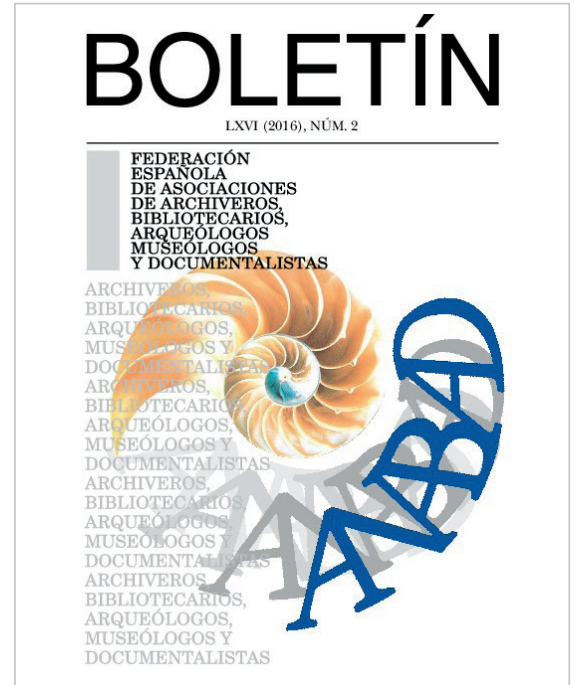

Portada de un número del Boletín de la Anabad https://www.anabad.org/category/publicacionesanabad/boletin

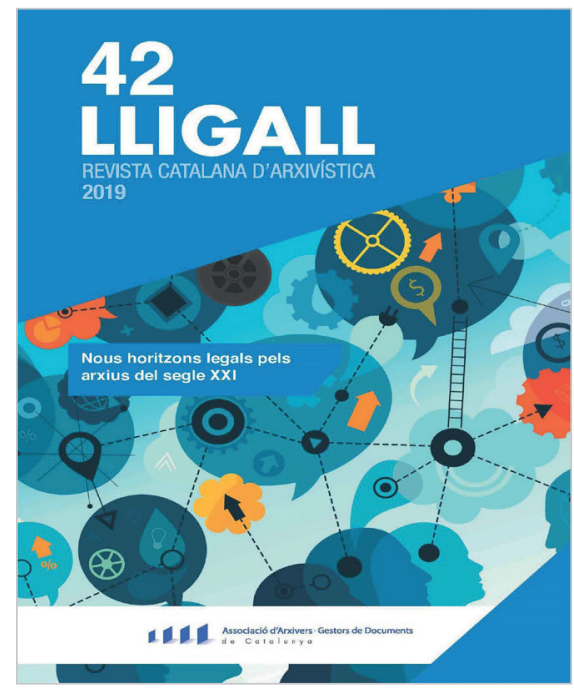

Portada de un número de la Revista Lligall https://arxivers.com/publicacions/revista-lligall 


\section{Boletín de la Anabad}

https://www.anabad.org/category/publicaciones-anabad/boletin/page/2

Publicada por la Federación Española de Asociaciones de Archiveros, Bibliotecarios, Arqueólogos, Museólogos y Documentalistas (Anabad), es la revista más antigua de las 3 estudiadas, publicada desde 1950. Es trimestral y presenta temas de interés para archiveros, bibliotecarios, museólogos y documentalistas. Aborda los problemas teóricos y prácticos de los profesionales representados por la Federación. La web de Anabad da acceso a todos los números del período de esta investigación. En algunos artículos hay resúmenes y palabras clave, pero en otros no; además, varios relacionados con el tema, no son artículos, sino presentaciones en formato ppt. En el portal Dialnet hay un sumario completo disponible de los años 2001 a 2018, pero no están todos los artículos completos. Para esta investigación, utilizamos las versiones impresas y en $C D$ disponibles en la biblioteca de la Facultad de Ciencias de la Documentación de la Universidad Complutense de Madrid.

Con el fin de establecer un análisis coherente de los artículos de las tres revistas, en vista del objetivo de investigación y el largo período que abarca, se crearon categorías de análisis basadas en títulos y resúmenes, y cuando estos no eran lo suficientemente claros y objetivos para identificar a qué categoría

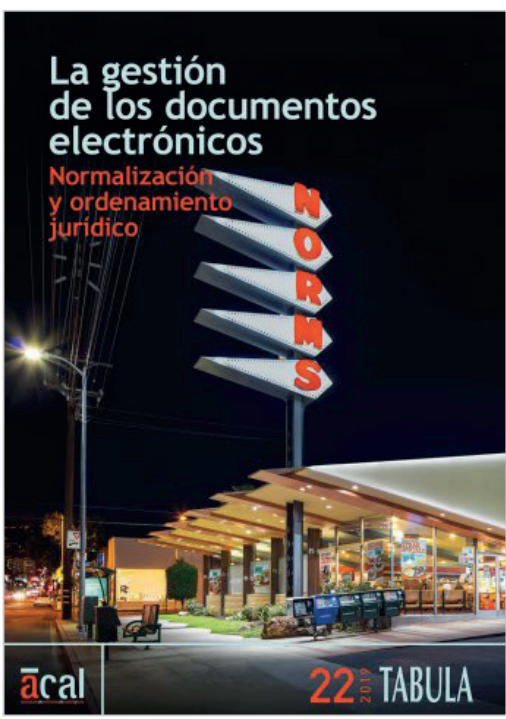

Portada de un número de la revista Tábula https://www.acal.es/index.php/tabula se ajustaban, se utilizó el texto completo para un análisis más preciso de su contenido. Se crearon 14 categorías iniciales, agrupadas posteriormente en 8 categorías intermedias, y por fin, reducidas a 4 categorías de análisis finales.

La categorización se realizó de acuerdo con reglas orientadoras para la elaboración y clasificación de categorías de análisis, que son, según Carlomagno y Caetano-da-Rocha las siguientes:

“- reglas claras de inclusión y exclusión;

- las categorías deben ser mutuamente excluyentes;

- cada una no ha de ser muy amplia, siendo homogénea en contenido;

- contemplar todo el contenido posible; la categoría "otro" debe ser residual;

- objetiva, no capaz de ser codificada de manera diferente dependiendo de la interpretación del analista". (Carlomagno; Caetano-da-Rocha, 2016, p. 173)

Las 4 categorías finales creadas para analizar los artículos, basadas en el análisis de los artículos mismos, son las siguientes:

- Relaciones entre archivos, archivística y tecnología digital: artículos que realizan una investigación exploratoria, sin proponer la aplicación de metodologías o presentar resultados prácticos;

- Influencias mutuas entre archivos, archivística y tecnología digital: artículos que presentan las influencias de la teoría, principios, conceptos, actividades y metodología de archivo en tecnología digital y viceversa;

- Retos teóricos y prácticos: artículos relacionados con la creación, preservación, acceso a documentos de archivo digital y necesidades de profesionales y usuarios;

- Relatos de experiencia (artículos que presentan y describen los usos de la tecnología digital en las actividades de archivo). Para aclarar el método de categorización utilizado, en la tabla 1 se muestran las categorías iniciales, intermedias y finales.

Tabla 1. Definición de categorías de análisis

\begin{tabular}{|c|c|c|}
\hline \multicolumn{3}{|c|}{ Tabla de definición de categorías } \\
\hline Categorías iniciales & Categorías intermedias & Categorías finales \\
\hline $\begin{array}{l}\text { 1. Principios y conceptos } \\
\text { 2. Enseñanza de archivística }\end{array}$ & $\begin{array}{l}\text { 1. Historia archivística } \\
\text { 2. Teoría, principios y conceptos } \\
\text { 3. Docencia e investigación }\end{array}$ & $\begin{array}{l}\text { 1. Relaciones entre archivos, archivística y } \\
\text { tecnología digital }\end{array}$ \\
\hline $\begin{array}{l}\text { 3. Acceso } \\
\text { 4. Adquisición } \\
\text { 5. Aplicación de conceptos de archivo en la } \\
\text { apropiación de herramientas tecnológicas. } \\
\text { 6. Aplicación de técnicas y tecnologías en el } \\
\text { área de archivos y archivística } \\
\text { 7. Clasificación } \\
\text { 8. Creación } \\
\text { 9. Descripción/indexación } \\
\text { 10. Difusión } \\
\text { 11. Valoración }\end{array}$ & $\begin{array}{l}\text { 4. Aplicación de tecnologías en la resolución } \\
\text { de problemas } \\
5 \text {. Gestión de documentos y funciones } \\
\text { archivísticas } \\
\text { 6. Políticas y sistemas de gestión de docu- } \\
\text { mentos electrónicos/digitales }\end{array}$ & $\begin{array}{l}\text { 2. Influencias mutuas entre archivos, archivís- } \\
\text { tica y tecnología digital }\end{array}$ \\
\hline 12. Conservación/ Preservación & 7. Preservación y seguridad de la información & 3. Retos teóricos y prácticos \\
\hline $\begin{array}{l}\text { 13. Presentación de experiencias, usos de } \\
\text { sistemas de gestión y software } \\
\text { 14. Digitalización }\end{array}$ & $\begin{array}{l}\text { 8. Tratamiento archivístico de documentos } \\
\text { electrónicos/digitales }\end{array}$ & 4. Relatos de experiencias \\
\hline
\end{tabular}




\section{Resultados}

Entre los artículos de las tres revistas, sólo fueron seleccionados aquellos cuyos contenidos están evidentemente vinculados con el tema de investigación, es decir, que interrelacionan los archivos, la archivística y la tecnología digital.

Algunos artículos tratan el tema de manera conjunta con museos y museología o biblioteca y biblioteconomía. En estos casos, aunque presenten una visión integrada de dos o más áreas, más allá de la archivística, fueron incluidos los artículos que tratan el tema de investigación específico.

Sin embargo, hay otros en los que el tema está relacionado con los archivos y la archivística, pero cubren superficial o genéricamente el tema de la tecnología digital; en este caso, no se incluyeron en el análisis. Aún más, los que tratan con documentos digitales desde el punto de vista de la ley o la informática, por ejemplo, pero sin establecer conexiones con los archivos o con la archivística, también fueron ignorados.

La tabla 2 presenta una síntesis cuantitativa de los resultados obtenidos para las tres revistas analizadas.

Tabla 2. Comparación cuantitativa entre las revistas Tábula, Lligall y Boletín la Anabad

\begin{tabular}{|c|c|c|c|}
\hline Revista & Tábula & Revista Lligall & Boletín de la Anabad \\
\hline Año de creación & 1992 & 1988 & 1950 \\
\hline $\begin{array}{l}\text { Años, según marco cronológico, en que se publi- } \\
\text { caron artículos sobre el tema }\end{array}$ & $\begin{array}{c}2002-2006^{2} \\
2008-2016 \\
2018\end{array}$ & $\begin{array}{l}2001-2012 ; \\
2014-2015\end{array}$ & $\begin{array}{c}2001 ; \\
2003-2008 ; \\
2011-2014 ; \\
2018\end{array}$ \\
\hline Cantidad de artículos & 240 & 241 & 463 \\
\hline Cantidad de artículos sobre el tema investigado & 66 & 52 & 59 \\
\hline
\end{tabular}

Tábula es la que contiene más artículos sobre el tema, con un $27,5 \%$. A continuación, la Revista Lligall presenta $21,57 \%$ y por último, el Boletín de la Anabad incluye el 12,74\%. En el total de artículos producidos por las tres revistas, el 75\% se ocupa del tema de los archivos, de la archivística y de la tecnología digital, lo que puede considerarse significativo teniendo en cuenta que las revistas no tratan exclusivamente de temas archivísticos y de temas sobre tecnologías, a excepción de algunos números especiales dedicados al tema, como los números 5 de 2002 y 17 de 2014 de Tábula, dedicados respectivamente a Documentos electrónicos y Ego archivo. Memorias personales en un mundo digital; así como el número 4 de 2008, del Boletín de la Anabad, dedicado a Memoria y tecnología. Esta última revista, que publica no sólo artículos, sino presentaciones de cursos realizados en eventos, hay varios cursos dedicados al tema ${ }^{3}$.

En cuanto a los años en que se publican los artículos con el tema analizado en esta investigación, se puede ver la distribución por revistas en los gráficos 1,2 y 3 .

En la revista Tábula se observa que, desde el punto de vista cronológico y en orden creciente, destacan los artículos sobre la materia de inves- 
tigación en los años 2002 (07), 2009 (10), 2014 (07) y 2016 (16). Una de las razones por las cuales se puede evidenciar la mayor ocurrencia en estos años es porque estos números están dedicados a los siguientes temas:

- Documentos electrónicos (2002);

- Archivos híbridos. Las transformaciones en materiales, procesos y productos (2009);

- Ego archivo. Memorias personales en un mundo digital (2014);

- Archivos smart: Hacia la gestión inteligente (2016).

No se publicaron artículos sobre el asunto en 2007 y 2017. O más bien, en 2007, el número está dedicado al tema Combates por la memoria. Archivística de la posmodernidad donde se aborda la cuestión, pero en una perspectiva bastante difusa, y no como el tema central de los artículos.

En la Revista Lligall ya hay una variación menor de artículos por año, excepto en 2010, donde aparecen 11 artículos, y como se ha expuesto en el caso de Tábula, hay un número dedicado al aspecto La preservació digital: de què parlem? El segundo año en el que acontecen más ocurrencias (4 artículos), es en 2008, cuando no hay referencia a un tema específico para ese número de la revista. No se identificaron artículos sobre la materia de investigación en los años 2005/1, $2013 / 1$ y $2013 / 2$.

En el Boletín de la Anabad hay mayor dispersión en el número de artículos por año sobre el asunto investigado. En orden cronológico creciente, los artículos sobre el tema de investigación se destacan en los años 2006/4 (04), 2008/4 (06), $2012 / 3$ (04) y 2013/3 (09). Se encontró la misma razón en esta revista que en otras: se realizaron las siguientes ediciones especiales de estos números:

- Ponencias y comunicaciones del VIII Congreso de Anabad (2006/4);

- Memoria y tecnología, congreso celebrado en Madrid, del 13 al 15 de febrero de 2008 (2008/4);

- un curso titulado Archivos y gestión de documentos ante la web semántica, que probablemente dio lugar a los artículos (2012/3);

- curso dedicado a Europeana (2013/3).

No se identificaron artículos sobre el tema de investigación en los años/trimestres 2001/1, 2001/3, 2001/4, 2002/1, 2002/2, 2002/3, 2002/4, 2003/1, 2003/2, 2004/1 e 2, 2004/4, 2005/1, 2005/2, 2006/1, 2009/2, 2009/3, 2010/1, 2010/2, 2010/3, 2010/4, 2011/1, 2011/3, 2011/4, 2012/2, 2013/1, 2013/2, 2013/4, 2014/3, 2014/4, 2015/2, 2015/4, 2016/1, 2016/3, 2016/4, 2017/1, 2017/2, 2017/3, 2017/4 у 2018/1.

Con respecto a la comunicación científica de cada una de las tres revistas, de acuerdo con las categorías de análisis creadas, se presentan los resultados en la tabla 3.

Tabla 3. Cantidad de artículos en las tres revistas de acuerdo con las cuatro categorías de análisis entre 2001 y 2018

\begin{tabular}{|c|c|c|c|c|}
\hline Categorías & Tábula & Lligall & Anabad & Total \\
\hline Relaciones entre archivos, archivística y tecnología digital & 15 & 0 & 5 & 20 \\
\hline Influencias mutuas entre archivos, archivística y tecnología digital & 36 & 29 & 23 & 88 \\
\hline Retos teóricos y prácticos & 9 & 15 & 4 & 28 \\
\hline Relatos de experiencias & 6 & 8 & 27 & 41 \\
\hline
\end{tabular}

En la categoría "Relaciones entre archivos, archivística y tecnología digital”, hay 20 artículos, la mayoría de Tábula. Tratan cuestiones más teóricas, por ejemplo, preservar la memoria en el mundo digital, participación de usuarios y activismo de archivo, cambios de paradigma de la disciplina y aspectos relacionados con los propios profesionales.

En la categoría "Influencias mutuas entre archivos, archivística y tecnología digital”, la mayoría de los artículos se centran en la aplicación de la tecnología en la resolución de problemas, la gestión de documentos y las funciones archivísticas, las políticas y el sistema de gestión de documentos digitales. Estos artículos abarcan desde la protección de datos y el derecho a olvidar en internet hasta el uso de tecnologías en la clasificación y descripción de archivos, el uso de la web para la difusión de archivos, etc. Hay una cierta proporcionalidad de esta categoría entre las revistas.

En la categoría "Retos teóricos y prácticos", que cubre los artículos relacionados con la preservación y seguridad de la información, hay 28 artículos desde diferentes perspectivas: sostenibilidad técnica y económica, creación y mantenimiento de metadatos, posibilidad de un vocabulario común sobre el tema...

Finalmente, la categoría de "Relatos de experiencias", con artículos sobre el tratamiento de documentos de archivo digitales, describiendo experiencias, usos de sistemas de gestión y software, y la digitalización, abarca 41 artículos en total. En este caso destaca la revista Boletín de la Anabad con mayor número.
El uso de la electrónica y la informática en la producción y gestión de los documentos, y en su acceso y recuperación, potencia la desaparición progresiva de la cultura del papel 
Con respecto a la autoría de los artículos por países (que no siempre coincide con la nacionalidad de los autores), la revista Tábula presenta contribuciones de 14 autores de siete países, además de España, a saber: Australia, Canadá, Estados Unidos, Países Bajos, Portugal, Suecia y Suiza. La Revista Lligall tiene 16 autores de cinco países además de España, como son: Bélgica, Croacia, Canadá, Estados Unidos y Portugal. El Boletín de la Anabad cuenta con la participación de 9 autores de tres países, además de España, que son: Brasil, Estados Unidos y Portugal.

En todas las revistas las autorías variaron de 1 a 6 autores por artículo.

\section{Conclusiones}

Teniendo en cuenta los resultados obtenidos en el análisis de estas revistas, aunque no es un estudio exhaustivo de toda la producción científica en el área de los archivos y la archivística española, se puede inferir que los subtemas que más han despertado el interés de la comunidad profesional y científica en España, en términos cuantitativos, están vinculados a la categoría que llamamos "Influencias mutuas entre archivos, archivística y tecnología digital”, que se puede decir que es la categoría que demuestra más claramente las conexiones, o intentos de unión, entre métodos, funciones y actividades típicas de los archivos (gestión de documentos, clasificación, descripción, difusión, acceso) y las posibilidades que ofrece la tecnología digital para realizarlos y ampliarlos.

Al mismo tiempo, se observa que en esta interfaz entre los archivos, la archivística y la tecnología digital, los procedimientos convencionales en el campo se enfrentan a nuevos modelos de lógica digital, lo que requiere que investigadores y profesionales discutan, prueben y experimenten con la versatilidad y complejidad cada vez mayores de los sistemas y soluciones automatizadas.

Si en el siglo XX se podía percibir cierta linealidad en la producción tecnocientífica española relacionada con el tema (descripción, y sus herramientas, gestión electrónica de los documentos y difusión), ahora, en el siglo XXI hay una intersección de reflexiones y dudas sobre los temas más diversos: servicios archivísticos, herramientas y redes sociales, alcance de la teoría y metodología archivísticas. O sea, como dijo Terry Cook (2012), no se trata simplemente de transponer conceptos y procedimientos archivísticos utilizados para documentos producidos y procesados en papel, sino de repensar la teoría y la práctica en un contexto de cambio social y tecnológico permanente.

En segundo lugar, hay relatos de experiencias que en su mayoría exponen el uso de la digitalización para la difusión y/o preservación de documentos de archivos o innovaciones promovidas por la tecnología digital que contribuyen a una mayor visibilidad de los archivos. Sin embargo, puede verse una variedad de acciones aisladas sin, con pocas excepciones, una reflexión sobre la calidad de la digitalización como técnica y proceso a largo plazo.

Las otras categorías, "Relaciones entre archivos, archivística y tecnología digital”, y "Retos teóricos y prácticos" están casi en el mismo nivel, aunque muy por detrás de las anteriores, mostrando también la atención de la comunidad científica y profesional española a los problemas que deben profundizarse con el proceso tecnológico, cada vez más dinámico y que afecta directamente al funcionamiento de las entidades públicas y privadas, y eso se refleja a su vez en la producción y el procesamiento de documentos, información y comunicación. 
Sin embargo, es digno de mención que se observa una tendencia de la archivística actual a experimentar las posibilidades tecnológicas para su desarrollo más que innovar en términos teóricos y epistemológicos. Para esto, nos parece que es necesario profundizar en el conocimiento ya obtenido por los archivos y por la archivística a lo largo de su existencia como una disciplina científica para enfrentar con audacia e imaginación el futuro. De acuerdo con Craig y Cook,

"Así como la identidad personal está anclada en un fuerte sentimiento histórico (...) lo mismo le ocurre a nuestra identidad profesional - ambas provienen de la capacidad de experimentar... una continuidad. Si no hay nada en el pasado en lo que valga la pena fijarse, y además, con orgullo, no hay nada en lo que podamos cifrar nuestras esperanzas de futuro" (Craig, 1992, p. 121 apud Cook, 1996, p. 1).

Cook, todavía refuerza,

"sin una continuidad con el pasado los rumbos del futuro carecen de legitimidad. Si no comprendemos los esfuerzos intelectuales de nuestros antecesores, perdemos el beneficio de sus experiencias" (Cook, 1996, pp. 1-2).

Y podemos agregar: sin el esfuerzo de comprender el mundo actual en que vivimos, inmerso en innovaciones tecnológicas, no podremos planificar el futuro y crear nuevos significados para la investigación científica y para la profesión de archiveros.

Tenemos la intención de verticalizar este estudio haciendo un análisis más detallado de los artículos, buscando identificar convergencias y divergencias teóricas y metodológicas archivísticas en vista de la tecnología digital disponible.

\section{Notas}

1. Véase: Gobierno de España. Punto de acceso general: tu punto de acceso a las administraciones públicas. https://administracion.gob.es/pag_Home/espanaAdmon/Administracion-electronica.html\#.XtVBTjozbIU

2. No se encontró el número referente al año 2001. La búsqueda fue realizada tanto en la web de ACAL como en la de Dialnet. Es posible que no fuera publicado el número de ese año.

3. El Boletín de la Anabad publicó presentaciones que no fueron consideradas para los propósitos de este estudio en:

- 2013, n. 4, Preservación digital: retos y propuestas actuales;

- 2014, n. 4, Patrimonio cultural en internet: balance y perspectivas;

- 2015, n. 4, Preservación digital y acceso continuo a la información.

\section{Referencias}

Carlomagno, Márcio C.; Caetano-da-Rocha, Leonardo (2016). “Como criar e classificar categorias para fazer análise de conteúdo: uma questão metodológica". Revista eletrônica de ciência política, v. 7, n. 1. https://doi.org/10.5380/recp.v7i1.45771

Cook, Terry (1996). "Interacción entre la teoría y la práctica archivísticas desde la publicación del manual holandés en 1898". En: Actas del XIII Congreso internacional de archivos. Beijing, China, 2-7 septiembre 1996. Madrid.

Cook, Terry (2012). "Arquivologia e pós-modernismo: novas formulações para velhos conceitos". Informação arquivística, v. 1, n. 1, pp. 123-148.

http://www.aaerj.org.br/ojs/index.php/informacaoarquivistica/article/view/9

Craig, Bárbara (1992). "Outward visions, inward glance: Archive history and professional identity". Archival issues, v. 17, n. 2, pp. 113-124.

https://www.jstor.org/stable/41101829?seq=1

España (2015). “Ley 39/2015, de 1 de octubre, del procedimiento administrativo común de las administraciones públicas". BOE, n. 236, 2 octubre.

https://www.boe.es/eli/es/l/2015/10/01/39/con

Gobierno de España (2020). Punto de acceso general: tu punto de acceso a las administraciones públicas. https://administracion.gob.es/pag_Home/espanaAdmon/Administracion-electronica.html

Sánchez-Mairena, Alfonso (2014). "Acercando los archivos a los ciudadanos. Una experiencia desde el Portal de Archivos Españoles (Pares) del Ministerio de Educación, Cultura y Deporte". Arbor, v. 190, n. 765, a103.

https://doi.org/10.3989/arbor.2014.765n1010 